\title{
Injectable Biologics for the Treatment of Degenerative Disc Disease
}

\author{
Ajay Matta ${ }^{1} \cdot$ W. Mark Erwin ${ }^{1,2}$ \\ Published online: 23 July 2020 \\ (C) The Author(s) 2020
}

\begin{abstract}
Purpose of Review Spinal pain and associated disability is a leading cause of morbidity worldwide that has a strong association with degenerative disc disease (DDD). Biologically based therapies to treat DDD face significant challenges posed by the unique milieu of the environment within the intervertebral disc, and many promising therapies are in the early stages of development. Patient selection, reasonable therapeutic goals, approach, and timing will need to be discerned to successfully translate potential therapeutics. This review provides a brief overview of the status of intradiscal biologic therapies.

Recent Findings Proposed systemic delivery of therapeutic agents has not progressed very much in large part due to the risk of adverse events in remote tissues plus the very limited vascular supply and therefore questionable delivery to the intervertebral disc nucleus pulposus. Intradiscal delivery of therapeutic proteins shows good potential for clinical trials and translation with encouraging results from large animal pre-clinical studies plus an enhanced understanding of the biology of DDD. There are a few cell-based therapies currently under pre-clinical and clinical trial investigation; however, these attempts continue to be hampered by unknown if any, mechanism of action, no downstream detection of transplanted cells, mixed results concerning efficacy, small sample numbers, and a lack of objective evidence of pain mediation.

Summary Treatment of DDD using biologically based therapeutics is a widely sought-after goal; however, potential therapies need to address pain and disability in larger, well-controlled studies.
\end{abstract}

Keywords Intervertebral disc disease $\cdot$ Back pain $\cdot$ Stem cell treatment $\cdot$ Disc injections

\section{Abbreviations}

AAV Adeno-associated virus

BMP-7 Bone morphogenetic protein-7

BMP-R Bone morphogenetic protein receptor

CTGF Connective tissue growth factor

DDD Degenerative disc disease

ECM Extracellular matrix

EGF Epidermal growth factor

This article is part of the Topical Collection on Updates in Spine Surgery-Techniques, Biologics, and Non-operative Management

W. Mark Erwin

mark.erwin@utoronto.ca

1 Notogen Inc, 661 University Ave., Suite 1300, Toronto, Ontario M5G 0B7, Canada

2 Divisions of Orthopedic and Neurological Surgery, University of Toronto, 661 University Ave., Suite 1300, Toronto, Ontario M5G 0B7, Canada

$\begin{array}{ll}\text { GDF-5/6 } & \text { Growth and differentiation Factor-5/6 } \\ \text { IGF } & \text { Insulin growth factor } \\ \text { IVD } & \text { Intervertebral Disc } \\ \text { NC } & \text { Notochordal cells } \\ \text { NF-kB } & \text { Nuclear Factor kappa B } \\ \text { NP } & \text { Nucleus pulposus } \\ \text { NPPCs } & \text { Nucleus pulposus progenitor cells } \\ \text { OP-1 } & \text { Osteogenic protein-1 } \\ \text { PDGF } & \text { Platelet-derived growth factor } \\ \text { PDGFR } & \text { Platelet-derived growth factor receptor } \\ \text { PRP } & \text { Platelet-rich plasma } \\ \text { rh } & \text { Recombinant human } \\ \text { CLCs } & \text { Chondrocyte-like cells } \\ \text { IL-1 } \beta & \text { Interleukin-1 beta } \\ \text { IL-6 } & \text { Interleukin-6 } \\ \text { IL-8 } & \text { Interleukin- } 8 \\ \text { TGF-1/3 } & \text { Transforming growth factor beta-1/3 } \\ \text { TGF } \beta R & \text { Transforming growth factor beta receptor } \\ \text { TNF } \alpha & \text { Tumor necrosis factor alpha }\end{array}$




\section{Introduction}

Back and neck pain secondary to degenerative disc disease (DDD) is the health condition with the highest economic cost to society, with direct and indirect costs estimated to range between $\$ 19.6$ and $\$ 118.8$ billion in the USA [1-3]. Epidemiologic studies have reported that spinal pain is the leading cause of years lost to disability worldwide with degenerative disc disease (DDD) accounting for a large proportion of back pain $[3,4]$. DDD results in the loss of cellularity, structural integrity, biomechanical properties, and height of the intervertebral disc that can contribute to pain, instability, and deformity [5]. Since there is no cure and there are controversial roles for surgery restricted to cases of instability or deformity, current treatments for DDD are limited to symptom management, such as physical therapies, antiinflammatory medications, and analgesics. The intervertebral disc (IVD) nucleus pulposus (NP) variably contains stem cells (nucleus pulposus progenitor cells or "NPPCs"), chondrocyte-like cells (CLCs), notochordal cells (NCs) (in youth in humans and in some animals such as rats, rabbits, and non-chondrodystrophic dogs), and fibroblasts, collectively termed "NP" cells. DDD is a multifactorial condition, but trauma, aging, genetics, and occupational stresses are factors that lead to a catabolic cascade of increased inflammatory cytokines and extracellular matrix (ECM) degrading molecules/enzymes resulting in excessive inflammation and progressive tissue damage $[5,6]$. The degenerative intervertebral disc (IVD) loses cellularity, structural integrity, and biomechanical properties and changes in nociception, biological changes that can variably contribute to pain, instability, and deformity [5]. Impaired biological regulation of the IVD as described above can lead to motor unit (the two vertebrae and disc lying in between) overload and secondary pain arising from inflammation/dysfunction of the segmental facet joints, joint capsules, and associated musculature [7]. Therefore, new interventions including biologics and/or tissue engineering approaches are currently under intense investigation with a view to being able to influence the course of the disorder [8].

\section{Therapeutic Interventions}

The intervertebral disc is largely avascular apart from the peripheral annulus, whereas the inner annulus and nucleus pulposus are hypoxic, ischemic, aneural, and isolated from the immune system. The IVD is sandwiched between the upper and lower vertebral end plates that, in addition to loading platforms, act to control the diffusion of nutrients, waste products, and gases principally from the IVD NP into and out of the vertebral body. It has been reported that progressive DDD involves calcification of the tiny pores within the endplate resulting in impaired diffusion and compromise of the gas/ nutrient exchange that in turn furthers the degenerative cascade $[9,10]$. Therefore, to be effective, any biological intervention must be able to mitigate the harsh, pro-inflammatory, pro-catabolic, and anti-anabolic environment within the degenerative disc.

\section{Systemic Therapies}

Oral or injectable therapeutics that may address DDD face considerable challenges in that they must first undergo absorption without degradation and then transport into the largely avascular, ischemic IVD. Interestingly, a number of manuscripts have been published detailing the use of systemic treatments for DDD such as the use of melatonin, Celastrol, or glucosamine sulfate [11-13]. In the melatonin study, the investigators reported that there may be a role played by melatonin in the development/progression of DDD in that pinealectomy has been shown to accelerate DDD in some vertebrates and that melatonin acts via specific cell surface receptors [11]. The conclusions of the study were that melatonin is a crucial regulator of NP cell function and that in vitro treatment of human IVD NP cells with melatonin downregulated extracellular matrix remodeling enzymes, increased collagen type 2 and aggrecan expression, and decreased cellular proliferation. The study demonstrated in vitro evidence that melatonin can influence NP cells and may provide a hypothetical model for how pinealectomy may influence DDD; this study was limited to an in vitro approach, and the translational potential for such therapy remains obscure. Another systemic approach to treat DDD has been published concerning the use of "Celastrol," a traditional Chinese medicine used in the treatment of a host of illnesses from diabetes to obesity, atherosclerosis, hearing loss, cancer, and neurodegenerative conditions [12]. The possible mechanism(s) of action appear to be principally via suppressing the pro-inflammatory pathway by inhibiting activation of NF- $\mathrm{KB}$ as well as mediating the activity of JNK kinases and caspase mediators of apoptosis [12]. Limiting the use of Celastrol is the low bioavailability and relatively narrow toxicity range of the drug as well as any reasonable route of administration. In the study by Chen et al., the drug was administered via intraperitoneal injection daily for up to 6-week post-needle puncture injury prior. Although the investigators report favorable anti-degenerative effects of treatment as well as favorable results using human IVD NP cells in vitro, the translational aspects of this kind of therapy are challenging. Systemic administration would require that the concentration of the agent must meet therapeutic levels within the hypoxic, ischemic, and avascular IVD without meeting systemic toxic levels. Amino sugars such as glucosamine sulfate/ hydrochloride have been postulated to confer benefit to degenerative joint disease such as osteoarthritis, with conflicting 
results. An in vivo study examining the use of oral glucosamine supplementation in a rabbit model of DDD showed that injured animals treated with oral glucosamine demonstrated an anti-anabolic effect [13]. The ability of any systemically applied intervention to treat DDD must overcome the challenges of the relatively avascular, ischemic, and isolated IVD NP with little progress over the past few decades, leading to rise of the intradiscal approach of direct injection of the therapeutic agent.

\section{Biomolecular Injection}

The direct injection of biomolecules into the IVD NP including anabolic/anti-catabolic proteins has been extensively studied particularly over the past decade. Growth factors encompass a broad range of pro-anabolic biomolecules that generally increase cellular proliferation, cellular viability, and beneficial effects upon the extracellular matrix. There are a multitude of growth factors known, some of which lie within "super families" such as the transforming growth factor superfamily (TGF). Within the overall umbrella of the TGF superfamily are the bone morphogenetic proteins (BMPs) (including BMP-2, BMP-7, BMP-12), as well as other BMP subfamilies like GDF-5/-6, as well as TGF- $\beta 1$ and TGF- $\beta 3$ among others. There are other biomolecules molecules including insulin-like growth factor (IGF), platelet-derived growth factor (PDGF), epidermal growth factor (EGF), as well as a poorly defined "cocktail" of factors contained within plateletrich plasma (PRP)[14]and/or catabolic enzyme inhibitors, all of which have been attempted as therapeutic interventions to treat DDD [8].

With respect to the delivery of growth factors, a study involving the injection of BMP-7, also known as OP-1, into a rabbit model of DDD was reported 16 years ago, and this procedure reportedly led to an increase in disc height with an improved elastic modulus [15]. Another study in which BMP2 was injected into degenerative rabbit discs led to worsened degeneration with enhanced vascularization and fibroblast proliferation [16]. However, a follow-up study using a canine model of DDD and an intradiscal administration of recombinant human (rh)BMP-7 not only did not show a therapeutic benefit, but it caused undesired bone formation external to the IVD [17]. These two reports underscore important aspects with respect to the injection of a putative therapeutic protein. The salient aspect concerning the injection of putative therapeutic agents is an understanding of the mechanisms of action of the growth factor and their respective signaling. For example, GDF-5/6, sub-classes of BMPs, themselves members of the TGF superfamily, signal through specific surface receptors that activate highly conserved intracellular signaling proteins, in this case, known as "Smads." BMPs signal via Smad-1, 5, and 8 whereas TGF- $\beta 1$ and TGF- $\beta 3$ signal via Smad-2, 3, and 4. These respective signaling cascades are of vital importance with respect to their downstream activation of dependent genes. The BMPs, by definition, are bone-inducing molecules that although also having proliferation potential and some beneficial aspects regarding extracellular matrix synthesis and also induce the formation of bone. This is the clear conclusion in the study involving injection of BMP-7 (OP-1) in the canine study. The Smad-2, 3, and 4 intracellular signaling pathways do not lead to bone formation but rather upregulate cell survival, ECM synthesis, and downregulation of proinflammatory signaling. Specifically, TGF- $\beta 1$ downregulates pro-catabolic signaling induced by pro-inflammatory cytokines as IL- $1 \beta, T N F \alpha$, IL- 6 , and IL- 8 , all of which are major players in progressive degenerative disc disease (Fig. 1).

These signaling events are tightly regulated and involve interplay with other mediators which in the case of BMPs include both canonical and non-canonical signaling that involve MAP kinases, Wnts, Akt/mTOR microRNAs, and importantly Runx 2 as possibly a key integrative signaling molecule [18]. The non-canonical pathways involve in Smadindependent signaling also implicate P38 MAP kinases that in turn can activate Runx2 that is central to osteoblastic differentiation and bone formation. It is vital to understand these signaling pathways to avoid undesirable bone formation as was seen following injection of BMP-7/OP-1 in the canine study cited above. TGF- $\beta 1$ on the other hand signals via the Smad-2/3/4 pathway that does not implicate Runx2 and osteogenesis [18]. In the studies published by Matta et al., there were impressive anti-degenerative effects and no reports of untoward bone formation [19, 20].

Published accounts have suggested that growth factor delivery would need to be repeated and have high doses of the therapeutic protein in order to provide any benefit $[8,21]$. Theoretically, activity of endogenous proteases within the IVD may degrade injected therapeutic proteins, and/or the persistence and bioavailability of injected biomolecules may be transient. However, recent published data refute this suggestion in that Matta et al. demonstrated in both rat and chondrodystrophic canines that a single injection of recombinant human connective tissue growth factor (rhCTGF) and recombinant human transforming growth factor beta-1 (rhTGF- $\beta 1$ ) suspended within an excipient solution showed ongoing transcription activity at least 14 weeks post-injection in canines as well as preservation of disc height and retained biomechanical properties compared with vehicle (saline) injections [20]. It is therefore unknown whether therapeutic protein delivery has only a transient activity particularly if the proteins are delivered within a formulation that enhances the bioavailability of the proteins. Some have advocated the use of slow release formulation or vector transmission in order to increase the duration of molecular delivery to the target tissue [8]. With respect to increasing the therapeutic effect of biomolecule delivery, Yan et al. investigated GDF-5 packaged in 


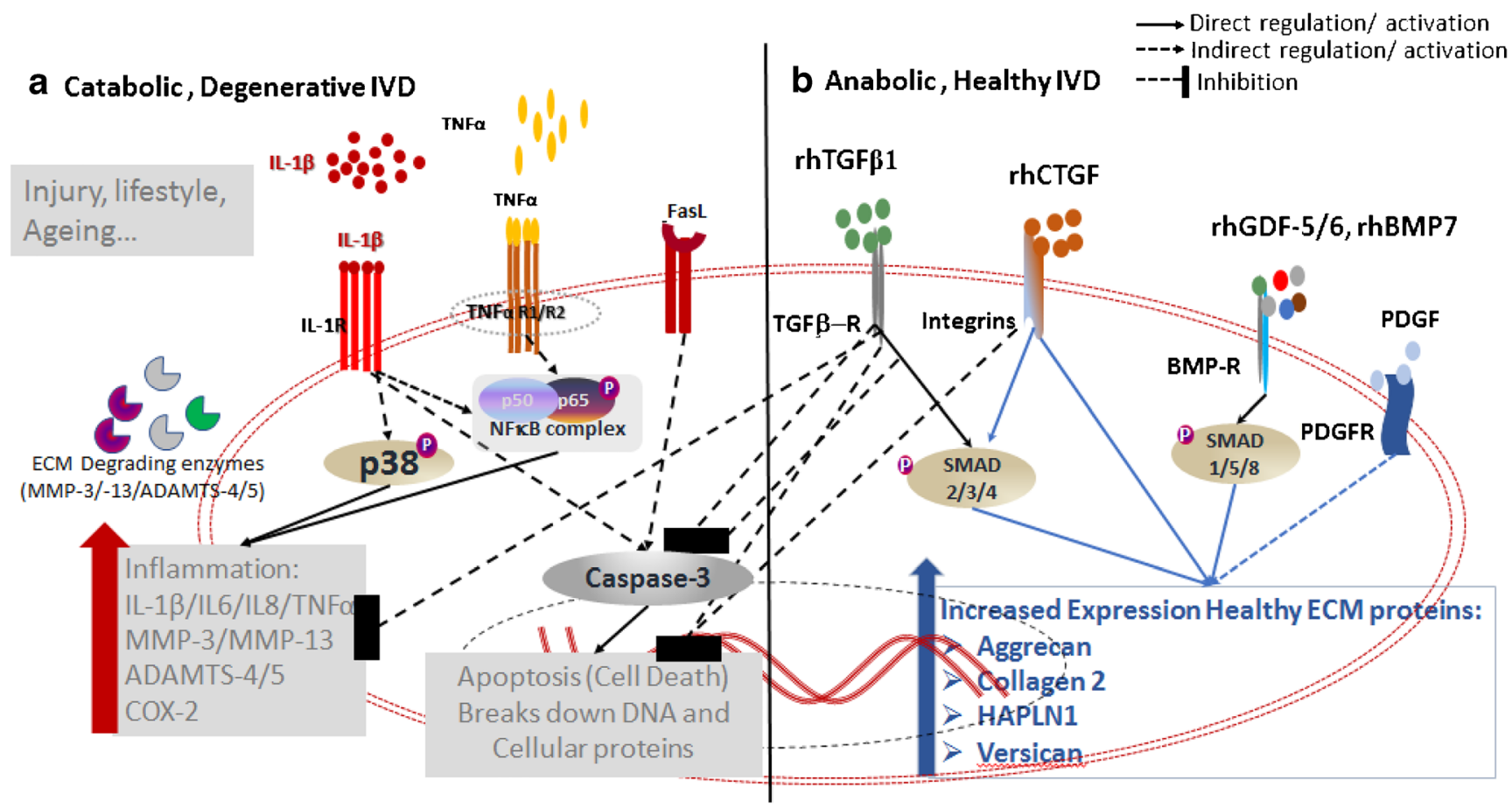

Fig. 1 Role of biologics therapy for degenerative disc disease (DDD). Schematic representing (a) pro-catabolic, degenerative microenvironment in IVD with increased expression of pro-inflammatory cytokines (IL-1 $\beta, \mathrm{TNF} \alpha$ ) and death ligand (FasL) because of injury, aging, or lifestyle changes. These pro-inflammatory cytokines activate nuclear factor kappa B (NF-kB) and p38MAPK signaling pathways inducing matrix metalloproteinases (MMP-3/MMP-13), aggrecanases (ADAMTS-4/5), pain-associated protein cyclooxygenase 2 (Cox2) in degenerative IVDs; (b) An intradiscal biologic therapy includes growth

microspheres that reportedly enabled the slow release of GDF5 for over 42 days. This approach reportedly demonstrated some improvement in a rat-tail model of DDD in terms of increased disc height, sulfated glycosaminoglycan content, and improved histological scores [22]. With respect to longer term bioactivity, Tellegen et al. recently published an account where celecoxib-loaded microspheres were injected in a preclinical canine model and reported that the release of celecoxib was observed for over 28 days; however, there was no meaningful regenerative effect [23]. Nonetheless, the notion of enhancing delivery of a biomolecular therapy using sustained, slow-release mechanisms may have utility if dose, concentration, and pharmacokinetics can be appropriately controlled and validated.

\section{Therapeutic Gene Delivery}

There have been numerous accounts of attempts to deliver genes that encode therapeutic proteins into the IVD to potentiate the presence of these proteins and mediate potential limitations of direct therapeutic protein delivery. Several possible vectors including adenovirus, adenoassociated virus (AAV), retrovirus, baculovirus, and factors (rhTGF $\beta-1 / 3$, rhCTGF, rhGDF-5/6, rhBMP-7, PDGF), PRP, or cellular replacement therapy. Treatment with a combination of rhTGFb1 and rhCTGF inhibits inflammation-induced signaling, thereby mitigating inflammation and inducing pro-anabolic, healthy ECM proteins (aggrecan, Collagens) both in vitro and in vivo animal models of DDD. Treatment with other growth factors including rhGDF-5/6, rhBMP-7, and PDGF has been reported to induce expression of healthy extracellular matrix proteins in IVD

lentiviruses have been investigated. Although some of these gene delivery systems have shown promising results in pre-clinical investigations, safety is an overriding concern [24*0]. Limitations to these approaches include the lack of integration of the adenoviral vector into the host DNA, which although reducing mutation risk in the protein expressed by the virus is expressed transiently. AAV only carries a limited amount of genetic information and carries with it difficulties in introduction into the target cells [25]. Nonetheless, therapeutic gene delivery faces challenges with safety due to a study using rabbits in which a higher dose of adeno-associated viral vectors expressing TGF $\beta 1$ and rhBMP-7 induced bilateral lower limb paralysis clearly indicating the potential risk of this approach [26]. Furthermore, undesirable transgene-induced protein production may lead to unintended adverse events indicating that the gene therapy approach as traditionally applied may provide more risk than benefit [27••]. With respect to genebased approaches, there have been recent attempts at the use of CRISPR-Cas9 gene editing to knock out and/or repair dysfunctional gene regulation. However, these approaches are very early and largely limited to in vitro experimentation $[28 \bullet \bullet]$. 


\section{Platelet-Rich Plasma}

Platelet-rich plasma (PRP) is a method whereby a small volume of concentrated activated platelets is injected into an area of injury and logically extends the idea of growth factor-based therapy since the mechanism of action of PRP is almost certainly due to the growth factor content of the injectate that can activate tissue repair $[29,30]$. In vitro experimentation has shown that PRP can stimulate cell proliferation and induce proteoglycan and glycosaminoglycan synthesis in soft tissue such as tendon and muscle [31]. A pre-clinical study using PRP in rat-tail models of DDD provided some evidence of anti-degenerative effects in the animals that received a PRP injection immediately, post injury, or 2 weeks later and showed some reduced degeneration; however, animals that received the injections 6 weeks post injury developed much more degenerative changes [32]. These results are interesting in that the rat disc is highly notochordal, and notochordal cells have been shown to confer anti-degenerative effects on IVD NP cells; therefore, it would be difficult to determine the beneficial effects of an acute injection of any therapeutic as compared with notochordal cell-based activity when the injection occurs within the acute or early sub-acute period. Matta et al. determined that the resolution of needle puncture injury matures by 10 weeks post injury resulting in significant loss of notochordal and stem cells in intervertebral disc nucleus pulposus $[19,20]$. Overall, there have been several studies evaluating in vitro and in vivo injection of PRP as a potential DDD therapy; however, there is considerable inconsistency using in vivo pre-clinical animal models [33]. A recent review by Akeda et al. has shown that although there have been in vitro and some in vivo (with mixed results) studies concerning PRP injections to treat DDD, only one doubleblind randomized controlled trial has been completed that showed significant improvements in functional rating scale, patient satisfaction, and numerical rating scale (NRS) worst pain at 8-week follow-up. The lack of no treatment control group beyond 8 weeks severely limits conclusions of this study. The authors reported that most of the treated patients were followed for 1 year with "beneficial effects" with respect to the FRI Index (measures participation perception of function and pain related to performing dynamic movements and holding static positions). Nonetheless, Oswestry disability or VAS scores were not reported [29, 34]. Surprisingly, no imaging studies were reported for the study patients. Also, only $56 \%$ of the patients reported being satisfied with the treatment. Potential confounders/limitations to the use of PRP are the lack of standardization of dose, large donor variability, method of preparation, and the lack of understanding with respect to purported mechanism of action. Although the study results are interesting, it is difficult to imagine a hypothetical mechanism for the reported improvements in only 8 weeks, and if "most of the treated patients" reported beneficial effects 1 year later also in the absence of imaging, one is left to wonder about the results with no control group beyond 8 weeks. Furthermore, with respect to the notion of intradiscal biotherapeutics, there has been explanation or even hypothesis concerning how the effects of a single PRP injection may confer long-term benefits or how pain relief as reported by Tuakli-Wosornu et al. may be conferred within 8 weeks.

\section{Cellular-Replacement Therapy}

In addition to the catabolic, pro-inflammatory changes that occur within the degenerative disc, DDD is also known to involve a loss of viable cells [35-37]. Therefore, cellular replacement strategies have emerged as potential methods to mitigate against the progressively acellular disc as a possible regenerative therapy. Several potential cell types have been postulated as candidates for cellular replacement including fibroblasts, bone marrow, adipose, umbilical cord-derived stem cells, IVD NP cells, or disc-derived chondrocytes. Some of these cellular replacement therapeutics have been and continue to be studied in clinical trials following limited pre-clinical studies. However, much remains to be determined concerning the use of cellular replacement.

Mechanism of Action There is scant evidence for the mechanism of action of transplanted cells given the wide variety of cells used for this purpose. For example, dermal-derived fibroblasts have been investigated as a potential source; however, in this approach, the desire is to alter the cellular configuration of the IVD into one of a connective tissue rather than confer a regenerative strategy. A recent publication concerning the use of dermal fibroblast as possible cellular transplants purported to show that such transplants conferred restorative effects upon Cynomolgus monkeys. In this study, six young monkeys that received surgical exposure and following needle puncture were either injected with dermal fibroblasts or sham controls (details lacking with respect to the sham procedure). The authors reported that the animals injected with the fibroblasts demonstrated retained IVD height as compared with needle puncture and hypothesize that the treatment resulted in a reparative fibrosis repair [38]. Of note, the fibroblast transplants occurred immediately at the time of disc injury with no time interval between needle puncture injury and cell therapy. Further, the treatment groups were only followed for 8 weeks, a very short period of time from which to draw meaningful conclusions. Another limitation to the study pertained to the phenotypic and TGF- $\beta$ and Smad signaling that the authors reported to be induced by fibroblasts. The authors used rat IVD NP cells co-cultured with fibroblasts and deduced that fibroblastic cells can induce NP cells to adopt a more fibroblastic phenotype [38]. Monolayer culture is known to induce fibroblastic differentiation, and rattail IVDs contain an almost $100 \%$ notochordal cellular 
phenotype, and normoxic $\left(21 \% \mathrm{O}_{2}\right)$ tissue culture is inconsistent with the intradiscal environment. Finally, the MRI data concerning the Cynomolgus monkey study had very broad variability and few numbers of animals leading to difficulty in arriving at convincing data.

Although not a cellular therapy, others have published with respect to injecting the degenerative disc with a formulation including lactic acid to induce a fibrous, connective tissue "healing." A single ascending dose study of the "STA363" compound has been completed in 15 patients with chronic discogenic low back pain (August 2019); however, no results have yet been posted (https://clinicaltrials.gov/ct2/show/ results/NCT03055845).

\section{Stem Cells}

One of the most studied sources of stem cells is bone marrow (stromal) cell-sourced stem cells (BMSCs) that have been the subject of numerous clinical trials. Historically, clinical trials involving BMSCs have been of small sample size and lack adequate controls and a continued lack of description of mechanism of action or evidence that the transplanted cells actually integrate into the NP [39]. A study published by Henriksson et al. concerning the traceability of mesenchymal stromal cells injected into the discs of only 4 patients resulted in all patients subsequently undergoing spinal fusion surgery [40••]. However, post-surgery, the IVD tissue removed was investigated for the presence of iron sucrose that was pre-labeled in the MSCs. The investigators were able to detect iron sucrose 8 months post-transplantation, and it was claimed that the cells had differentiated into chondrocyte-like cells [40••]. It is significant in that this study is among the only trials that demonstrate the presence of cells post-transplant over the longer term; however, all subjects progressed to require spinal fusion surgery post-transplant, yielding the questionable utility of such transplants in the mitigation of DDD, let alone any regenerative capacity. Other clinical trials involving the injection of up to $25 \times 10^{6} \mathrm{BMSCs}$ or between 6 and $18 \times 10^{6}$ cells yield further evidence that there is no consensus with respect to the number of cells that ought to be transplanted (https:// clinicaltrials.gov/ct2/show/record/NCT01860417), (https:// clinicaltrials.gov/ct2/show/NCT01290367).

\section{Conclusions}

Over the past decade, considerable progress has been in our understanding of the mechanisms involved with the development and progression of DDD. These advances have led to the evolution of novel candidate therapies and, in some cases, clinical trials; however, none have yet achieved the desired goal that convincingly modulating the course of DDD. Among the obstacles posed to biological therapy for the degenerative disc are the reduced $\mathrm{pH}$, avascular status, chronic inflammation, and progressive cell death that occur with DDD. Overcoming these challenges will require a minimally invasive intervention that can activate repair by endogenous IVD NP cells with or without cellular replacement that is active over the longer term. Most importantly, the therapy will need to positively impact pain of discogenic origin, a challenge that has a constellation of driving forces. It is therefore necessary to understand the contributions of biological factors that drive discogenic pain, which along with other patientspecific determinants summate in the full clinical picture. To this end, it is also important to keep expectations of what intradiscal therapy might realistically achieve at the forefront in the development of these yet to come innovative therapeutics.

\section{Compliance with Ethical Standards}

Conflict of Interest Ajay Matta $\mathrm{PhD}$ declares no conflict of interest. He receives salary support from Notogen Inc.

W Mark Erwin DC PhD declares no conflict of interest. He receives salary support from the University of Toronto, Canadian Memorial Chiropractic College, and Notogen Inc.

Human and Animal Rights and Informed Consent This article does not contain any studies with human or animal subjects performed by any of the authors.

Open Access This article is licensed under a Creative Commons Attribution 4.0 International License, which permits use, sharing, adaptation, distribution and reproduction in any medium or format, as long as you give appropriate credit to the original author(s) and the source, provide a link to the Creative Commons licence, and indicate if changes were made. The images or other third party material in this article are included in the article's Creative Commons licence, unless indicated otherwise in a credit line to the material. If material is not included in the article's Creative Commons licence and your intended use is not permitted by statutory regulation or exceeds the permitted use, you will need to obtain permission directly from the copyright holder. To view a copy of this licence, visit http://creativecommons.org/licenses/by/4.0/.

\section{References}

Papers of particular interest, published recently, have been highlighted as:

- Of importance

- Of major importance

1. Katz JN. Lumbar disc disorders and low-back pain: socioeconomic factors and consequences. J Bone Joint Surg Am. 2006;88(suppl 2): 21-4.

2. Hoy $\mathrm{D}$, et al. The global burden of low back pain: estimates from the Global Burden of Disease 2010 study Ann Rheum Dis, 2014.

3. Vos T, et al. Years lived with disability (YLDs) for 1160 sequelae of 289 diseases and injuries 1990-2010: a systematic analysis for 
the Global Burden of Disease Study 2010. Lancet. 2013;380(9859): 2163-96.

4. Cheung KM, et al. Prevalence and pattern of lumbar magnetic resonance imaging changes in a population study of one thousand forty-three individuals. Spine. 2009;34(9):934-40.

5. Adams MA, Roughley PJ. What is intervertebral disc degeneration and what causes it? Spine. 2006;31:2151-61.

6. Freemont AJ. The cellular pathobiology of the degenerate intervertebral disc and discogenic pain. Rheumatology. 2009;48:5-10.

7. Panjabi Manohar M. A hypothesis of chronic back pain: ligament subfailure injuries lead to muscle control dysfunction. Eur Spine J. 2006;15:668-76.

8. Moriguchi Y, Alimi M, Khair T, Manolarakis G, Berlin C, Bonassar LJ, et al. Biological treatment approaches for degenerative disk disease: a literature review of in vivo animal and clinical data. Global Spine J. 2016;6(05):497-518

9. Rajasekaran S, Venkatadass K, Naresh BJ. Pharmacological enhancement of disc diffusion and differentiation of healthy, ageing and degenerated discs. Eur Spine J. 2008:17:626-43.

10. Nosikova Y, et al. Characterization of the annulus fibrosusvertebral body interface: identification of new structural features. J Anat. 2012;221(6):577-89.

11. Zheng L, et al. Melatonin inhibits nucleus pulposus cell proliferation and extracellular matrix remodeling via the melatonin membrane receptors mediated PI\#K-Akt pathway. J Pineal Res. 2017;63:e12435.

12. Jian C, et al. Celastrol reduces IL-1B induced matrix catabolism, oxidative stress and inflammation in human nucleus pulposus cells and attenuates rat intervertebral disc degeneration in vivo. Biomed Pharmacother. 2017;91:208-19.

13. Lloydine $\mathrm{J}$, et al. Glucosamine supplementation demonstrates a negative effect on intervertebral disc matrix in an animal model of disc degeneration. Spine. 2013;38:984-90.

14. Masuda K. Biological repair of the degenerated intervertebral disc by the injection of growth factors. Eur Spine J. 2008;17(Suppl 4): S441-51.

15. Miyamoto K, Masuda K, Kim JG, Inoue N, Akeda K, Andersson GBJ, et al. Intradiscal injections of osteogenic protein-1 restore the viscoelastic properties of degenerated intervertebral discs. Spine J. 2006;6(6):692-703.

16. Huang K-Y, Yan JJ, Hsieh CC, Chang MS, Lin RM. The in vivo biological effects of intradiscal recombinant human bone morphogenetic protein-2 on the injured intervertebral disc: an animal experiment. Spine. 2007;32(11):1174-80.

17. Nicole W, et al. Intradiscal application of rhBMP-7 does not induce regeneration in a canine model of spontaneous intervertebral disc degeneration. Arthritis Res Ther. 2015;17(137).

18. Shaifur RM, et al. TGF-B/BMP signaling and other molecular events: regulation of osteoblastogenesis and bone formation. Bone Res 2015:3.

19.• Matta A, et al. Molecular therapy for degenerative disc disease: clues from secretome analysis of the notochordal cell-rich nucleus pulposus. Sci Rep. 2017;7:45623. This manuscript detailed the first ever comprehensive analysis of proteins secreted by the non-chondrodystrophic canine nucleus pulposus and demonstrated that injection of notochordal cell-rich conditioned medium was able to mediate degeneration in a needle punctureinjured IVD NP. Signaling analysis and bioasay demonstrated that this regenerative effect was re-capitulated by the injection of Recombinant Human Connective Tissue Growth Factor and recombinant human Transforming Growth Factor Beta-1.

20.• Ajay M, et al. NTG-101: A novel molecular therapy that halts the progression of degenerative disc disease. Sci Rep. 2018;8:16809. This manuscript followed the same group's publication in 2017 and demonstrated in rat-tail, chondrodystrophic canine models of DDD and human IVD NP cells, that administration of a novel, molecular therapy 'NTG-101' consisting of Recombinant Human Connective Tissue Growth Factor and recombinant human Transforming Growth Factor Beta-1 plus excipients prevented the development or progression of DDD. The paper also demonstrates that IVDs injected with NTG-101 maintained disc height, biomechanical properties and robustly downregulated the expression of pro-inflammatory cytokines and inflammatory proteins within the IVDs.

21. Walsh AJ, Bradford DS, Lotz JC. In vivo growth factor treatment of degenerated intervertebral discs. Spine. 2004:29(2):156-63.

22. Yan J, Yang S, Sun H, Guo D, Wu B, Ji F, et al. Effects of releasing recombinant human growth and differentiation factor-5 from poly (lactic-co-glycolic acid) microspheres for repair of the rat degenerated intervertebral disc. J Biomater Appl. 2014;29(1):7280 .

23. Tellegen AR, Rudnik-Jansen I, Beukers M, Miranda-Bedate A, Bach FC, de Jong W, et al. Intradiscal delivery of celecoxibloaded microspheres restores intervertebral disc integrity in a preclinical canine model. J Control Release. 2018;286:439-50.

24.• Yoshiki T, Takashi Y, Kotaro N. Gene therapy approach for invertebral disc degeneration: an update. Neurospine. 2020;17:314. In this manuscript, Takeoka et al provide a review of various approaches to introduce genes encoding for the production of therapeutic proteins into the intervertebral disc. The underlying hypothesis that IVD NP cells can be engineered to modulate their secretion of therapeutic proteins and mTOR signaling and therefore inhibit undue autophagy and suppress degenerative disc disease is the underlying theme.

25. Woods BI, Vo N, Sowa G, Kang JD. Gene therapy for intervertebral disk degeneration. Orthop Clin N Am. 2011;42(4):563-74.

26. Wallach Corey J, et al. Saftey assessment of intradiscal gene transfer: a pilot study. Spine J. 2006;6:107-12.

$27 . \bullet$ Kang James D. Commentary on "gene therapy approach for intervertebral disc degeneration: an update". Neurospine. 2020;17:15-6. This commentary provides context to the manuscript by Takeoka et al by briefly reviewing the origins of gene therapy dating back to the early 1990 's and the initial enthusiasm of the approach. Also, Kang reviews important limitations of gene therapy in light of safety concerns for the patient and provides insight with respect to the notion of using RNA interference to inhibit pathways known to be implicated in the development and progression of degenerative disc disease.

$28 . \bullet$ Farhang N, et al. Lentiviral CRISPR epigenome editing of inflammatory receptors as a gene therapy strategy for disc degeneration. Hum Gene Ther. 2019;30:1161-74. In this manuscript, Farhang et al describe the feasibility of using CRISPR technology to to edit the expression of TNFreceptor 1 and the IL-1 receptors in human IVD NP cells in vitro. They report that this technology was able to downregulate TNF receptor signaling, however it did not result in alterations in the IL-1 receptor system. The authors hypothesize that although CRISPR can induce down regulation of TNF receptors, the technology has limitations with respect to other inflammatory pathways such as the IL receptors.

29. Koji A, et al. Platelet-rich plasma in the management of chronic low back pain: a critical review. J Pain Res. 2019;12:753-67. The publication by Koji et al provides a timely review of the use of platelet rich plasma (PRP) in the treament of back pain presumably secondary to DDD. The review encompasses the theory that the injection of releasate obtained from activated platelets (growth factors and an ill-defined combination of other molecules) into the intervertebral disc will somehow stimulate healing. The review details that there has only been one randomized, placebo controlled clinical study with a small number of patients an no control group beyond 8-weeks yet there was some perceived benefit in some patients. The manuscript 
further details that there is no convincing mechanism of action of 'PRP' in large part due to the heterogenity of production and individual variability. Large scale studies are necessary to determine if PRP is better than standard of care.

30. Shuji $\mathrm{O}$, et al. Effect of autologous platelet-rich plasma-releasate on intervertebral disc degeneration in the rabbit anular puncture model: a preclinical study. Arthritis Res Ther. 2012;14:R241.

31. Hudgens JL, et al. Platelet-rich plasma activates proinflammatory signlaing pathways and induces oxidtive stress in tendon fibroblasts. Am J Sports Med. 2016;8:1931-40.

32. Gullung Gregory B, et al. Platelet-rich plasma effects on degenerative disc disease: analysis of histology and imaging in an animal model. Evid Based Spine Care J. 2011;2(3):13-8.

33. Monfett M, Harrison J, Boachie-Adjei K, Lutz G. Intradiscal platelet-rich plasma (PRP) injections for discogenic low back pain: an update. Int Orthop. 2016;40(6):1321-8.

34. Tuakli-Wosornu Yetsa A, et al. Lumbar intradiskal platelet-rich plasma (PRP) injections: a prospective, double blind, randomized controlled study. Phys Med Rehabil. 2016;8:1-10.

35. Takashi Y, et al. Notochordal cell disappearance and modes of apoptotic cell death in a rat tail static compression-induced disc degeneration model. Arthritis Res Ther. 2014;16:R:31.

36. Cui LY, et al. IL-1beta sensitizes rat intervertebral disc cells to Fas ligand mediated apoptosis in vitro. Acta Pharmacol Sin. 2007;28: 1671-6.

37. Park JB, Lee JK, Park SJ, Kim KW, Riew KD. Mitochondrial involvement in fas-mediated apoptosis of human lumbar disc cells. J Bone Joint Surg Am. 2005;87:1338-42.

38. Chen C, Zhou T, Sun X, Han C, Zhang K, Zhao C, et al. Autologous fibroblasts induce fibrosis of the nucleus pulposus to maintain the stability of degenerative intervertebral discs. Bone Res. 2020;8.

39. Pennicooke B, et al. Biological treatment approaches for degenerative disc disease: a review of clinical trials and future directions. Cureus, 2016;(11).

$40 . \bullet$ Barreto HH, et al. The traceability of mesenchymal stromal cells after injection into degenerated discs in patients with low back pain. Stem Cells Dev. 2019;28:1203-11. This manuscript details an attempt to track mesenchymal stromal cells after transplantation into the intervertebral disc of only four patients living with painful degenerative discs. What was apparent in the study was that three of the four patients all went on to have spinal fusion surgery within 28 months post transplantation; therefore casting considerable doubt upon the benefit of transplantation. However, disc material obtained from the patients after surgery demonstrated the presence of pre-labeled iron sucrose within their intervertebral discs showing that some transplanted cells survived. The authors conclude that some of the transplanted cells differentiated into chondrocyte-like cells and that the transplanted cells and/or their daughter cells (if any) formed clusters within the IVD NP. Although the presence of some transplanted MSCs could ostensibly be found within the disc, the purpose of the transplanted cells-to treat degenerative disease must be concluded to have failed since three of the four cases progressed to require spinal fusion surgery.

Publisher's Note Springer Nature remains neutral with regard to jurisdictional claims in published maps and institutional affiliations. 